\title{
Linfoma não-Hodgkin bilateral do seio cavernoso como manifestação inicial da síndrome de imunodeficiência adquirida: relato de caso
}

\author{
Bilateral cavernous sinus non-Hodgkin's lymphoma as the presenting sign of \\ acquired immunodeficiency syndrome: case report
}

Alan Kardec Barreira Junior ${ }^{1}$, Frederico Castelo Moura ${ }^{2}$, Mario Luiz Ribeiro Monteiro ${ }^{2}$

\section{RESUMO}

Relato de caso com acometimento bilateral do seio cavernoso causado por linfoma não-Hodgkin em um paciente com síndrome da imunodeficiência adquirida. Paciente de 51 anos infectado pelo vírus da imunodeficiência adquirida há dois anos, sem terapia antirretroviral, apresentou-se com acometimento dos Ve VI nervos cranianos bilateralmente. Exame de tomografia computadorizada de órbitas mostrou-se dentro da normalidade. Como a hipótese diagnóstica principal era afecção do ápice orbitário ou do seio cavernoso, foi realizado exame de imagem por ressonância magnética que evidenciou lesão compatível com linfoma acometendo os seios cavernosos. Apesar da raridade dos linfomas primários do sistema nervoso central, essa condição deve ser considerada entre os diagnósticos diferenciais nos pacientes imunodeprimidos que apresentem oftalmoplegia e sinais radiológicos sugestivos de lesão infiltrativa do seio cavernoso.

Descritores: Seio cavernoso; Linfoma; Sistema nervoso central; Diplopia; Síndrome de imunodeficiência adquirida; Relatos de casos

\begin{abstract}
Case report of bilateral cavernous sinus syndrome due to primary non-Hodgkin lymphoma of the central nervous system in a patient infected by the human immunodeficiency virus. A 51-year-old male patient infected by the human immunodeficiency virus but without antiretroviral treatment developed paralysis of the V and VI cranial nerves. Imaging studies were obtained to investigate an orbital apex and a cavernous sinus syndrome. A computerized tomography scan of the orbit was normal but a highresolution magnetic resonance imaging demonstrated bilateral enlargement of the cavernous sinus. Although primary lymphoma of the central nervous system is a rare condition, it should be considered in the differential diagnosis in immunocompromised patients who develop ocular motility abnormalities and imaging signs suggestive of infiltrative cavernous sinus lesions.
\end{abstract}

Keywords: Cavernous sinus; Lymphoma; Central nervous system; Diplopia; Acquired immunodeficiency syndrome; Case reports

\section{INTRODUÇÃO}

Linfoma maligno primário no sistema nervoso central (SNC) é raro, mas a incidência vem aumentando nas últimas décadas devido ao crescente número de pessoas imunodeprimidas (p.ex: síndrome da imunodeficiência adquirida (SIDA) e transplantes de órgãos)(1). As manifestações clínicas da doença neurológica secundária ao linfoma maligno dependem das estruturas acometidas que incluem parênquima cerebral, leptomeninges, vasculatura cerebral e os nervos cranianos e periféricos ${ }^{(2-3)}$.

A síndrome de seio cavernoso (SSC) é definida pelo acometimento de dois ou mais nervos constituintes que são: III, IV, V (ramo frontal e maxilar), $\mathrm{VI}$ e fibras simpáticas ${ }^{(4)}$. Existem poucos relatos de linfomas malignos primários do SNC se apresentando inicialmente com SSC, geralmente relacionado à invasão do tumor localizado nos seios paranasais ou na base do crânio ${ }^{(4-5)}$.

Neste trabalho revisamos a literatura e relatamos um caso de linfoma primário do sistema nervoso central nos seios cavernosos como manifestação inicial da SIDA.

\section{RELATO DE CASO}

Paciente do sexo masculino, 51 anos, com diagnóstico de infecção pelo vírus da imunodeficiência adquirida (HIV) há dois anos, sem trata- mento antirretroviral, foi admitido em janeiro de 2007 no Hospital das Clínicas da Universidade de São Paulo com queixa de dor periorbitária esquerda e parestesia na hemiface ipsilateral seguidos de diplopia homônima ao olhar para esquerda após quatro dias. Após uma semana, os mesmos sintomas se estenderam para o lado direito. Negava em ambos os olhos (AO) perda da acuidade visual, blefaroptose, dor ocular, cefaléia ou obscurecimento visual transitório. Associada ao quadro neuroftalmológico, apresentou tumoração na axila direita um mês após o início da dor periorbitária, sem sinais inflamatórios. Nessa ocasião, a tomografia computadorizada de órbitas e crânio encontrava-se dentro dos limites da normalidade.

Ao exame oftalmológico inicial apresentava acuidade visual 1,0 (na escala decimal) em AO. Ao exame da motilidade ocular extrínseca havia paresia do músculo reto lateral bilateralmente, mais acentuada à direita (Figura 1) e os demais músculos apresentavam função normal. A sensibilidade corneana e da fronte estavam reduzidas à direita ao exame com monofilamento. A biomicroscopia do segmento anterior, a exoftalmometria com exoftalmômetro de Hertel, os reflexos pupilares e a fundoscopia foram normais em AO.

Aos exames laboratoriais, verificou-se leucopenia (2.500 leucócitos,com $46 \%$ de neutrófilos e $42 \%$ de linfócitos), acometimento renal (creatinina: 4,0 mg/dl e uréia: $69 \mathrm{mg} / \mathrm{dl}$ ) e hepático (TGP: $130 \mathrm{U} / \mathrm{L}$, fosfatase alcalina: 376 UI e gama GT: 452 UI) e hipoproteinemia
Submitted for publication: July 14, 2009

Accepted for publication: June 24, 2010

Study carried out at the Departamento de Oftalmologia e Otorrinolaringologia, Faculdade de Medicina, Universidade de São Paulo - USP - São Paulo (SP), Brasil.

Physician, Departamento de Oftalmologia e Otorrinolaringologia, Faculdade de Medicina, Universidade de São Paulo - USP - São Paulo (SP), Brasil.

2 Physician, Serviço de Neuroftalmologia e Órbita, Hospital das Clínicas, Faculdade de Medicina, Unysician, Serviço de Neuroftalmologia e Orbita, Hospital
Funding: No specific financial support was available for this study.

Disclosure of potential conflicts of interest: A.K.Barreira Junior, None; F.C.Moura, None; M.L.R.Monteiro, None.

Correspondence address: Frederico Castelo Moura. Rua Pedroso Alvarenga, 1.077 - São Paulo SP - 04531-012 - Brazil - E-mail: fredcastelo@terra.com.br

Editorial Note: After completing the confidential analysis of the manuscript, $A B O$ discloses, with his agreement, the name Dr. Luis Eduardo Morato Rebouças de Carvalho as a reviewer. We thank his effort and expertise in participating in this process. 

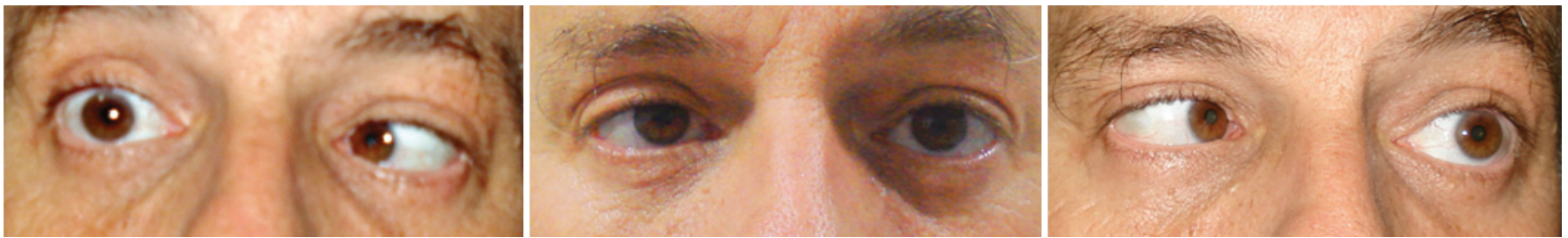

Figura 1. Motilidade ocular extrínseca apresentava paresia reto lateral bilateral.
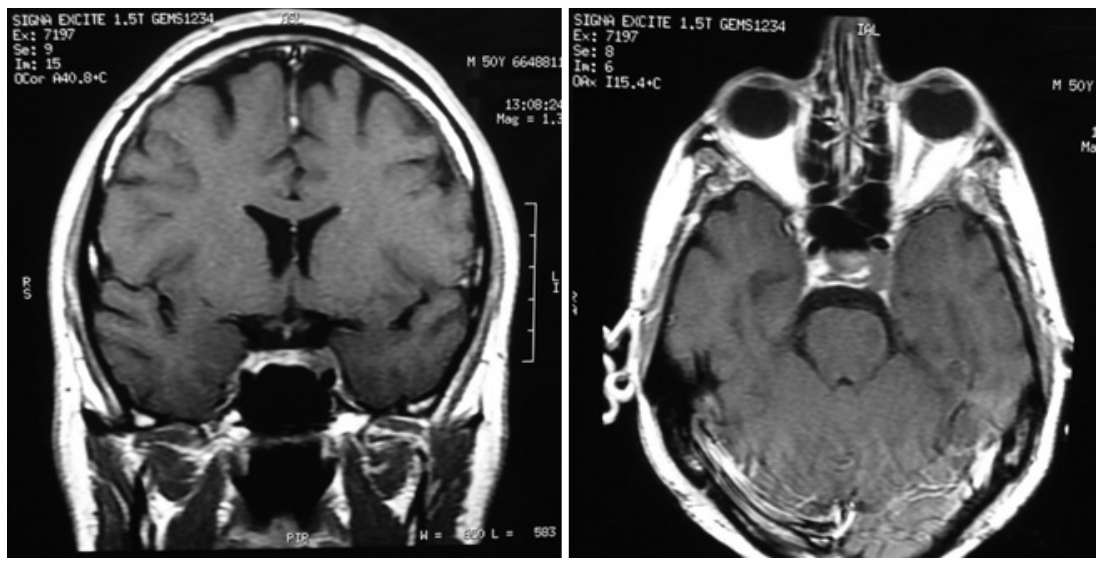

Figura 2. Imagem por ressonância magnética enfatizando T1 com contraste mostrando lesão isointensa com discreto realce no seio cavernoso bilateralmente.

(proteínas totais: 4,8 g/dl, albumina: 2,1 g/dl e globulinas 2,7 g/dl). A imagem por ressonância magnética (IRM) enfatizando T1 evidenciou lesão isointensa, com discreto realce após administração de gadolínio localizada em ambos os seios cavernosos, mais evidente à esquerda (Figura 2). Devido à suspeita de lesão neoplásica, foi realizado estudo histopatológico da lesão axilar que evidenciou hiperplasia linfóide atípica e a biópsia da medula óssea evidenciou infiltração de células linfóides fortemente sugestiva de linfoma não-Hodgkin. Algumas semanas após o diagnóstico, o paciente desenvolveu falência renal e hepática.

\section{DISCUSSÃO}

A SSC caracteriza-se pelo acometimento de pelo menos dois dos pares cranianos que o atravessam: III, IV, V (ramos frontal e maxilar), $\mathrm{VI}$ e fibras oculossimpáticas. Segundo Keane ${ }^{(6)}$, as principais causas de SSC, excluindo a etiologia traumática pós cirúrgica, são: tumores, afecções vasculares, infecções e inflamações, com repercussão unilateral na maioria dos $\operatorname{casos}^{(6)}$. A afecção bilateral dos seios cavernosos é rara e nestes casos predominam as causas vasculares, entre elas tromboflebite e malfomações arteriovenosas, principalmente fístula corotídeo-cavernosa ${ }^{(4)}$. A SSC secundária a linfoma do sistema nervoso central é extremamente rara e poucos casos semelhantes foram descritos na literatura ${ }^{(4,7)}$. Assim, o caso relatado neste trabalho apresentou etiologia e lateralidade incomuns para a SSC.

Em pacientes com infecção por HIV, as doenças que cursam com manifestação clínica neurológica mais frequentemente são: toxoplasmose, tuberculose do sistema nervoso central, leucoencefalopatia multifocal progressiva, doenças cerebrovasculares e aspergilose ${ }^{(8)}$. Javaloyas de Morlius et al. ${ }^{(4)}$ descreveram um caso similar ao nosso que evoluiu para óbito devido à disseminação sistêmica do linfoma não-Hodgkin ${ }^{(4)}$. O autor ressalta a importância da busca de manifestações extraneurológicas que permitam o diagnóstico por meio de procedimentos minimamente invasivos com baixa morbidade, como em nosso paciente a partir da biópsia da tumoração axilar. Arimoto et al. ${ }^{(1)}$ relataram caso sem antecedentes pessoais de morbidades com SSC. Sorologias para HIV, vírus Epstein Barr, hepatites $B$ e $C$ eram negativas. IRM evidenciava lesão tumoral invadindo seio cavernoso direito. Antes da ressecção cirúrgica, as principais hipóteses diagnósticas eram meningioma e neurinoma do trigêmeo devido à raridade de linfoma primário no SNC. O exame histopatológico revelou linfoma não-Hodgkin. Após 15 meses da ressecção cirúrgica, a paciente mantinha-se sem sinais de recorrências ou disseminação sistêmica do tumor ${ }^{(1)}$. Huisman et al ${ }^{(9)}$ descreveram um caso de linfoma primário de seio cavernoso na infância que evoluiu para óbito após três meses do diagnóstico ${ }^{(9)}$.

Nosso paciente se apresentou de forma rara com acometimento bilateral dos constituintes do seio cavernoso como manifestação inicial de SIDA, apesar da tomografia computadorizada sem alterações, o que ressalta a importância do exame neuro-oftalmológico adequado e o exame de ressonância magnética para confirmação do diagnóstico. Desta forma, linfoma não-Hodgkin deve ser considerado entre os diagnósticos diferenciais nos pacientes com HIV-AIDS e alteração de motilidade ocular extrínseca associada a sinais radiológicos sugestivos de acometimento no seio cavernoso.

\section{REFERÊNCIAS}

1. Arimoto H, Shirotani T, Nakau H, Hashizume K, Sakai Y, Matsukuma S. Primary malignant lymphoma of the cavernous sinus-case report. Neurol Med Chir (Tokyo). 2000;40(5): 275-9.

2. Bhatti MT, Schmalfuss IM, Eskin TA. Isolated cranial nerve III palsy as the presenting manifestation of HIV-related large B-cell lymphoma: clinical, radiological and postmortem observations: report of a case and review of the literature. Surv Ophthalmol. 2005; 50(6):598-606.

3. Coppeto JR, Monteiro ML, Cannarozzi DB. Optic neuropathy associated with chronic lymphomatous meningitis. J Clin Neuroophthalmol. 1988;8(1):39-45.

4. Javaloyas de Morlius M, Martínez Yélamos S, Huerta Villanueva M, Martínez Yelamos A. [Bilateral cavernous sinus syndrome due to non-Hodgkin's lymphoma as the presentation of HIV infection]. Med Clin (Barc). 2001;116(2):78-9. Spanish.

5. Williams Z, Norbash A, Goode RL. Cavernous sinus syndrome caused by a primary paranasal sinus non-Hodgkin's lymphoma. J Laryngol Otol. 1998;112(8):777-8.

6. Keane JR. Cavernous sinus syndrome. Analysis of 151 cases. Arch Neurol. 1996;53(10):967-71.

7. Ceyhan M, Erdem G, Kanra G, Kaya S, Onerci M. Lymphoma with bilateral cavernous sinus involvement in early childhood. Pediatr Neurol. 1994;10(1):67-9.

8. Sacktor N. The epidemiology of human immunodeficiency virus-associated neurological disease in the era of highly active antiretroviral therapy. J Neurovirol. 2002;8 Suppl 2:115-21. Review.

9. Huisman TA, Tschirch F, Schneider JF, Niggli F, Martin-Fiori E, Willi UV. Burkitt's lymphoma with bilateral cavernous sinus and mediastinal involvement in a child. Pediatr Radiol. 2003; 33(10):719-21. 